\title{
Normality of the Stock Index Futures of China
}

\author{
Ning Wang1*, Yibo Chen ${ }^{2}$, Bo Wang ${ }^{3}$ \\ ${ }^{1}$ Department of Information Management, School of Management, Shanghai University, Shanghai, China \\ ${ }^{2}$ Shanghai Liangyou Asset Management Company Limited, Shanghai, China \\ ${ }^{3}$ College of Urban Rail Transportation, Shanghai University of Engineering Science, Shanghai, China \\ Email: *ningwang@shu.edu.cn, chenyibo1029@gmail.com, wangbo519888@163.com
}

How to cite this paper: Wang, N., Chen, Y.B. and Wang, B. (2018) Normality of the Stock Index Futures of China. Journal of Mathematical Finance, 8, 86-101. https://doi.org/10.4236/jmf.2018.81007

Received: December 7, 2017

Accepted: January 28, 2018

Published: January 31, 2018

Copyright $\odot 2018$ by authors and Scientific Research Publishing Inc. This work is licensed under the Creative Commons Attribution International License (CC BY 4.0).

http://creativecommons.org/licenses/by/4.0/

\begin{abstract}
In this paper, we test the null hypothesis that the prices of stock index futures of China follow a random-walk process. Five hypothesis tests are applied to test the random-walk hypothesis (RWH). Each test uses both inter-day and intra-day returns. Compared with inter-day analysis, test results on intra-day data can describe the movements of intra-day markets more effectively, because intra-day analysis eliminates overnight news propagation, thus generating more precise conclusions on the intra-day market for intra-day traders. Three out of the five tests reject the RWH, whereas the other two cannot reject the RWH. Overall, the market is not fully efficient.
\end{abstract}

\section{Keywords}

China' Stock Market Random-Walk Hypothesis Statistical Test

\section{Introduction}

It has been studied for several decades that whether there exist laws that capture stock price movements, or the opposite, stock markets are efficient. Fama published his ground-breaking work on efficient market hypothesis (EMH) in 1970 [1]. Fama proposed three forms of efficiency, namely, weak-form, semi-strongform, and strong-form efficiency. Weak-form efficiency denotes that future prices cannot be predicted by analyzing past prices. Investors cannot earn excess returns in the long run by trading strategies based on historical share prices or other historical data. Semi-strong-form efficiency asserts that stock prices adjust to publicly available new information very rapidly and in an unbiased manner. Semi-strong-form efficiency implies that neither fundamental analysis nor technical analysis can consistently generate excess returns. Strong-form efficiency implies that stock prices have reflected all of the information, private or public, 
and no one can earn excess returns. Strong-form efficiency is impossible when legal barriers prevent information from being publicized. Fama argues that the primary role of financial markets is resource allocation; security prices at any time "fully reflect" all available information. Thus, firms can make production-investment decisions and investors can select their securities that represent the ownership of firms. Fama defines an "efficient" market as a market where stock prices always "fully reflect" the available information.

The EMH is a key assumption when developing the models and theories of financial markets; it has implications on the profitability of various forms of analysis. Moreover, the EMH has been extensively studied and debated in the academic community. Those who believe in the EMH contend that all of the public information has been reflected in the current stock price. Thus, any attempt to forecast the future stock price based on past information is doomed to fail. However, the financial crises in 2007 to 2012 have induced renewed scrutiny and criticism of the hypothesis.

The possibility of market inefficiency and subsequent profit opportunities appear to portfolio managers and investors. Possibilities exist for investors to determine undervalued stocks at low prices in an inefficient market, such that investors can buy the stocks and make money. From a broader perspective, if a stock market is inefficient, then the pricing mechanism does not efficiently allocate capital within an economy, with negative effects for the overall economy. The issue of how efficiently markets incorporate information into security prices is central to investors and policymakers who deal with equity markets. Specifically, this paper focuses on one form of market efficiency, that is, the empirical validity of random-walk hypothesis (RWH) in security markets. The RWH asserts that prices follow random walks, and price changes can be modeled as independently distributed random variables; in other words, in a given time point, the size and direction of the subsequent price change are random with respect to the current available knowledge.

A large amount of literature has tested the RWH of various markets through various statistical tests. When testing the RWH, all works use the returns (one representation of price changes) calculated from the spreads of every two consecutive closing prices. This type of returns is typically called inter-day returns. This paper inherits the routine of testing inter-day returns; intra-day returns are considered as well. Intra-day returns are calculated as the spreads of closing and opening prices on every day divided by the opening price of that day. Intra-day returns are more suitable for intra-day strategy developers because the effects of rolling days and overnight information are eliminated.

This paper aims to test the null hypothesis that the stock index futures prices of China follow a random-walk process. If the hypothesis cannot be rejected, then no trading strategy can generate the expected positive returns. Otherwise, the inefficiency induces profitability. Five hypothesis tests are applied to test the RWH. Each test uses both inter-day and intra-day returns. The fifth test deploys 
the idea of variance-ratio test and incorporates high-frequency data into the model. Three out of the five tests reject the RWH, whereas the other two cannot reject the RWH. Overall, the market is not fully efficient.

The contribution of this paper lies in three aspects. First, we use inter-day returns to test the RWH, in order to eliminate the overnight news propagation. Second, we deploy the idea of variance-ratio to test the RWH. Third, we find that the Chinese market is not fully efficient.

The remainder of this paper is organized as follows. Section 2 presents a survey on the random walk tests of worldwide markets. Section 3 performs five regular tests on the RWH of the stock index futures of China. Section 4 discusses the indications of the inefficacy of the Chinese market. Section 5 concludes this paper.

\section{Literature Review}

Considerable research has examined the efficiency of stock market price formation. The empirical evidence for the validity of RWH is mixed.

The seminal work of Lo and MacKinlay [2] tested the RWH for weekly stock market returns by comparing the variance estimators derived from the data sampled at different frequencies. The empirical results indicate that the random walk model is generally inconsistent with the stochastic behavior of weekly returns, particularly for the smaller capitalization stocks.

Researchers who support the RWH through statistics include Buguk and Brorsen [3], who tested the Istanbul Stock Exchange using its composite, weekly closing prices of industrial and financial indices. The results indicate that all three series are random walks, but a non-parametric test provides some evidence against random walks. Araújo Lima and Tabak [4] tested the RWH for China, Hong Kong and Singapore and revealed that the A-shares of the Chinese stock exchanges and the Hong Kong equity markets are weak-form efficient. However, Singapore and the B-shares of Chinese stock exchanges do not follow the RWH, which suggests that liquidity and market capitalization may explain the results of weak-form efficiency tests. Al-Khazali et al. [5] revisited the empirical validity of the RWH in eight emerging markets in the Middle East and North Africa (MENA) using a nonparametric variance ratio test. After correcting for measurement biases caused by thin and infrequent trading prevalent in nascent and small stock markets, Al-Khazali et al. cannot reject the RWH for the MENA markets. Borges [6] reported the RWH tests of stock market indices of France, Germany, United Kingdom, Greece, Portugal and Spain, and pointed out convincing evidence that monthly prices and returns follow random walks in all six countries. Borges [7] reported the results of four tests on the weak-form market efficiency applied to the PSI-20 index prices of the Lisbon stock market from January 1993 to December 2006. Borges demonstrated mixed evidence; however, the overall results suggested that the Portuguese stock market index has been approaching a random-walk behavior since 2000, with a decrease in the serial 
dependence of returns. Malkiel [8] argued that professional investment managers, both in the United States and abroad, do not outperform their index benchmarks, which provides evidence that large market prices do seem to reflect all of the available information.

Aside from statistical methods, Gersdorff and Bacon [9] analyzed the effect of US company M\&A announcements on the risk-adjusted rates of returns of stock prices. Their result supported semi-strong market efficiency, together with a positive signal that was exhibited by the sample of acquiring firms.

A major strand of literature employs a wide array of statistical tools for opposing the EMH. Gallagher and Taylor [10] outlined a simple macro model with overlapping wage contracts to investigate how the temporary and permanent components of stock price movements are related to aggregate macro-economic supply and demand disturbances. They revealed that the temporary component in U.S. stock prices is statistically significant, which supports that stock prices are not pure random walks. Lai et al. [11] examined the daily stock prices for the Kuala Lumpur Stock Exchange Composite Index from January 1977 to December 1999 and reported that prices behave in a non-random manner. Hoque et al. [12] reexamined the RWH for eight emerging equity markets in Asia, namely Hong Kong, Indonesia, Korea, Malaysia, the Philippines, Singapore, Taiwan, and Thailand. The hypothesis is tested with two new variance ratio tests. Hoque et al. contended that the stock prices of the eight Asian countries do not follow random walk with the possible exceptions of Taiwan and Korea. Balsara et al. [13] used the variance ratio test to reject the RWH for A- and B-shares stock market indices traded on the Shanghai and Shenzhen stock exchanges. Lim et al. [14] investigated the empirical validity of the weak-form EMH in the Mongolian equity market by four statistical tests. All of the tests presented results that were consistent with weak-form inefficiency.

Particularly, Azad et al. [15] pointed out the existence of market-wide trading-induced manipulations in the Bombay, Dhaka, and Karachi stock exchanges. Obtaining profitable volume-driven manipulations in the presence of market efficiency is impossible; thus, the presence of market manipulations suggests the inefficiency of South Asian markets.

Another stream of research contradicts random walk theory by predicting the market movements or devising trading strategies that generate excess earnings. Huang et al. [16] investigated the predictability of financial movement direction with support vector machine by forecasting the weekly movement direction of NIKKEI 225 index. The proposed forecasting model outperforms the random walk model. Peng and Xiong [17] modeled the process of distilling information by investors and examined its effect on asset-price dynamics. Their model demonstrated new cross-sectional implications for return predictability. Qian and Rasheed [18] investigated the predictability of the Dow Jones Industrial Average to demonstrate that not all periods are equally random. The Hurst exponent was used to select a period with significant predictability. Based on techniques from 
artificial intelligence, a prediction accuracy of up to $65 \%$ was achieved.

This study focuses on the normality of the stock index futures of China. The recent introduction of index futures has induced only a few studies. In addition, most studies are based on daily or weekly returns; this research classifies daily returns into intra-day returns and inter-day returns, and subsequently examines the two types of returns. Furthermore, this research proposes a new variant of the variance-ratio test that is based on high-frequency data (obtaining prices every half-second).

\section{Random Walk Tests}

This section tests the observed distribution of prices of CSI 300 index futures contracts. The CSI 300 index is a capitalization-weighted stock market index designed to replicate the performance of top 300 stocks traded in the Shanghai and Shenzhen stock exchanges. The nominal value of a contract is derived by multiplying the points of CSI 300 by 300 RMB. The basic information of futures contracts is summarized in Table $1^{1}$.

As shown in Table 1, four contracts (i.e., current month, next month, and next two calendar months) are traded concurrently. In this study, the main contract is examined. The main contract is the contract with the largest open interest among the four contracts. A large open interest indicates increased activity and liquidity of the contract. A noteworthy issue of the main contract is the

Table 1. Contracts of the CSI 300 stock index futures.

\begin{tabular}{|c|c|}
\hline Underlying & CSI 300 index \\
\hline Contract multiplier & $300 \mathrm{RMB}$ \\
\hline Unit & Index point \\
\hline Tick size & 0.2 point \\
\hline Contract months & $\begin{array}{l}\text { Monthly: current month, next month, next } \\
\text { two calendar quarters (four contracts in total) }\end{array}$ \\
\hline Trading hours & $\begin{array}{l}09: 15-11: 30,13: 00-15: 15 ; 09: 15-11: 30 \\
13: 00-15: 00 \text { (last trading day) }\end{array}$ \\
\hline Limit up/down & $\begin{array}{l}+/-10 \% \text { of settlement price on the } \\
\text { previous trading day }\end{array}$ \\
\hline Margin requirement & $12 \%$ of the contract value \\
\hline Last trading day & $\begin{array}{l}\text { Third Friday of the contract month, postponed to } \\
\text { the next business day if it falls on a public holiday }\end{array}$ \\
\hline Delivery day & Third Friday, same as "Last Trading Day" \\
\hline Settlement method & cash settlement \\
\hline Transaction code & IF \\
\hline Exchange & China Financial Futures Exchange \\
\hline Inception date & April 16, 2010 \\
\hline Capital requirement & $500,000 \mathrm{RMB}$ \\
\hline
\end{tabular}

${ }^{1}$ http://www.c_ex.com.cn/en_new/sspz/hs300zs/. 
calculation of inter-day returns. Assume that the closing prices of day $d$ and $d-1$ are denoted by $c_{d}$ and $c_{d-1}$, respectively, and the daily return of day $d$ are calculated as $r_{d}=\left(c_{d}-c_{d-1}\right) / c_{d-1}$. As the main contract changes at the end of a delivery day, $c_{d}$ and $c_{d-1}$ may refer to different contracts; day $d$ in such a case is called a rolling day.

We collected the opening and closing prices of index futures main contracts automatically from a public available platform. The time range is from April 16, 2010 to February 13, 2014. We collected 923 opening and closing prices. In the variant of variance-ratio test, we use tick $(500 \mathrm{~ms})$ price data and the data is bought from a company.

Applying statistical models to test the distribution of recorded data is highly common. In various financial theories, price changes (returns) are assumed to follow normal distributions. Therefore, we test the distribution of intra-day and inter-day returns. Intra-day and inter-day returns are calculated by the spread between opening and closing prices within one day and the spread between two closing prices, respectively. Since a total of 923 opening and closing prices are found, we have 923 intra-day returns. Due to the existence of rolling days, we delete 46 rolling days and obtain 877 inter-day returns.

\subsection{Jarque-Bera Test}

The Jarque-Bera test, which examines how the kurtosis and skewness of tested data effectively match a normal distribution, is used to investigate the validity of a normal assumption. The calculation equation is as follows:

$$
\begin{gathered}
J B=\frac{1}{6}\left[S^{2}+\frac{1}{4}(K-3)^{2}\right] \\
K=\frac{\frac{1}{n} \sum_{i=1}^{n}\left(x_{i}-\bar{x}\right)^{4}}{\left[\frac{1}{n} \sum_{i=1}^{n}\left(x_{i}-\bar{x}\right)^{2}\right]^{2}} \\
S=\frac{\frac{1}{n} \sum_{i=1}^{n}\left(x_{i}-\bar{x}\right)^{3}}{\left[\frac{1}{n} \sum_{i=1}^{n}\left(x_{i}-\bar{x}\right)^{2}\right]^{\frac{3}{2}}}
\end{gathered}
$$

where $n$ is the number of observations in the sample and $\bar{x}$ is the sample mean. The observations in this paper are the inter-day and intra-day returns. The null hypothesis is a joint hypothesis of the skewness being zero and the excess kurtosis being zero. Samples from a normal distribution have an expected skewness of zero and an expected excess kurtosis of zero, which is the same as a kurtosis of three.

Table 2 shows the results of the Jarque-Bera test on the index futures. The pvalues of intra-day and inter-day tests are smaller than 0.05 ; therefore, the null hypothesis is rejected at a significance level of $95 \%$. The movement of index futures price is not a random walk, and profitability exists. 
Table 2. Results of the Jarque-Bera test.

\begin{tabular}{cccccccc}
\hline Code & Return type & $\bar{x}$ & variance & $K$ & $S$ & JB statistic & p-value \\
\hline $22^{\star} \mathrm{IF}$ & Intra-day & -0.00034 & 0.00021 & 5.60202 & 0.05962 & 261.21300 & $<1 \mathrm{E}-15$ \\
& Inter-day & -0.00041 & 0.00021 & 5.53576 & -0.01441 & 234.99600 & $<1 \mathrm{E}-15$ \\
\hline
\end{tabular}

\subsection{Runs Test}

This section deploys a runs test to verify the serial independence of the returns. The runs test is a non-parametric test. Run pertains to a series of increasing values or a series of decreasing values, (i.e., a successive sequence with the same sign). The number of increasing (or decreasing) values is the length of the run. For example, in a series of,,,,,,++---++4 positive signs and 3 negative signs are present, and the number of runs is 3 .

The first step in a runs test is to count the number of runs in the data sequence. The literature provides several means of defining runs; however, the formulation in all of the cases must generate a dichotomous sequence of values. In this section, runs are defined in two forms. The first form defines returns larger than (less than) zero by positive (negative) signs. The second form defines relative returns larger than (less than) zero by positive (negative) signs, where relative returns are real returns minus the average of all returns. The second form of runs removes the effect of trend in the testing period. The first and second forms of runs are called absolute and relative forms, respectively.

In a random data set, the probability that the $(i+1)$ th value is larger or smaller than the ith value follows a binomial distribution, which forms the basis of the runs test. Under the null hypothesis that a two-valued data sequence is random, the number of runs $n_{r}$ in a sequence of $n$ elements is a random variable whose conditional distribution given the observation of $n_{+}$positive values and $n_{-}$negative values is approximately normal, with

$$
\begin{gathered}
\text { mean } \mu_{r}=\frac{2 n_{+} n_{-}}{n}+1 \\
\text { variance } \sigma_{r}=\sqrt{\frac{2 n_{+} n_{-}\left(2 n_{+} n_{-}-n\right)}{n^{2}(n-1)}} \\
Z=\frac{n_{R}-\mu_{R}}{\sigma_{R}} \approx N(0,1)
\end{gathered}
$$

These parameters do not assume that the positive and negative elements have equal probabilities of occurring, but only assume that the elements are independent and identically distributed. If the number of runs is significantly higher or lower than expected, the hypothesis of statistical independence of the elements may be rejected. Theoretically, if the returns of two consecutive trading days have positive (negative) correlation, fewer (more) runs will be observed.

Table 3 shows the results of runs test for different configurations, classified by the type of return type and form of runs. The z-statistics of all four configurations are smaller than 1, which indicates that RWH cannot be rejected by this 
Table 3. Results of runs test.

\begin{tabular}{cccccccc}
\hline Return type & Runs form & $n_{+}$ & $n_{-}$ & $\mu_{r}$ & $\sigma_{r}$ & $n_{r}$ & z-statistic \\
\hline Inter-day & Absolute & 396 & 481 & 435.381 & 14.6595 & 441 & 0.383312 \\
Inter-day & Relative & 415 & 462 & 438.241 & 14.7561 & 447 & 0.792604 \\
Intra-day & Absolute & 421 & 502 & 458.946 & 15.0652 & 467 & 0.567811 \\
Intra-day & Relative & 436 & 487 & 461.091 & 15.1358 & 471 & 0.833324 \\
\hline
\end{tabular}

test. The test results, to some extent, infer the difficulty in determining a profitable trading strategy for daily returns. In addition, the z-statistics of intra-day type are larger than those of inter-day type, reconfirming that the price change within one day is less random than the overnight change.

\subsection{Augmented Dickey-Fuller (ADF) Test}

This section applies an ADF test to verify the existence of a unit root. If the return of index futures follows a random walk, then the time series should have a unit root. The following regression is estimated for the inter-day return series of index futures:

$$
\begin{aligned}
\Delta Y_{t} & =\alpha+\beta t+\gamma Y_{t-1}+\sum_{i=1}^{k} \theta_{i} \Delta Y_{t-i}+\varepsilon_{t} \\
& =\log \frac{c_{t}}{c_{t-1}}=\alpha+\beta t+\gamma \log c_{t-1}+\sum_{i=1}^{k} \theta_{i} \log \frac{c_{t-i}}{c_{t-i-1}}+\varepsilon_{t}
\end{aligned}
$$

where $Y_{t}=\log c_{t}$ is the $\log$ of daily closing price $c_{t}, \alpha, \beta, \gamma$ and $\theta_{i}$ are parameters, $\Delta$ represents first difference, $k$ is the number of lagged terms, and $\varepsilon_{t}$ is white noise that is normally distributed with a mean of zero. The $t$-test statistic for the null hypothesis of a random walk is $\gamma=0$ versus the alternative of $\gamma \neq 0$.

Table 4 shows the results of the ADF test on inter-day returns. "Level" and "First" denote the series itself and its first difference, respectively. The rows "with trend" and "without trend" present with and without the assumption of $\beta=0$. The result indicates that one unit root of the time series cannot be rejected while the first difference of the series under consideration is stationary, which confirms that the series is likely to be $I(1)$, and thus meets the necessary condition for a random walk.

Next, a time series representing intra-day returns is devised for the ADF test. Closing prices contain intra-day and overnight drifts, which are shown as the first and second fractions on the right side of Equation (1).

$$
c_{d}=\frac{C_{d}}{o_{d}} \frac{o_{d}}{o_{d-1}} c_{d-1}
$$

where $o_{d}$ and $c_{d}$ are the opening and closing prices on day $d$.

As the major consideration is the intra-day drift, only prices driven by intraday drifts are retained. Let $\hat{c}_{d}$ denotes the price driven by intra-day drifts, 
Table 4. Results of the ADF test on inter-day returns.

\begin{tabular}{ccc}
\hline Model & T-statistic & p-value \\
\hline Level & & \\
without trend & -2.1157 & 0.2386 \\
with trend & -2.8782 & 0.1701 \\
First & & \\
without trend & -31.1456 & $<0.0001$ \\
with trend & -31.1286 & $<0.0001$ \\
\hline
\end{tabular}

as the closing price of the first trading day is purely driven by the intra-day drift, therefore $\hat{c}_{1}=c_{1}$; on the second day, the price driven by the intra-day drifts of the first two days is $\hat{c}_{2}=\hat{c}_{1} \frac{C_{2}}{O_{2}}$; Similarly, $\hat{c}_{3}$ driven by intra-day drifts of the first three days is $\hat{c}_{3}=\hat{c}_{2} \frac{c_{3}}{O_{3}}$. Generally, the closing price $\hat{c}_{d}$ driven by intraday drifts equals

$$
\hat{c}_{d}=\hat{c}_{d-1} \frac{c_{d}}{o_{d}}
$$

In the $\mathrm{ADF}$ test, $Y_{t}$ is $\log \hat{c}_{d}$. Table 5 shows the results of the intra-day ADF test. With a significance level of $5 \%$, the result indicates that one unit root of the time series cannot be rejected while for the first difference of the series, the hypothesis can be rejected. With a significance level of $10 \%$, the null hypothesis is rejected for the intra-day returns with trend.

\subsection{Single Variance-Ratio Test}

This section presents the single variance-ratio test. The idea of this test is that if a series $Y_{t}$ follows a random walk process, then the variance of $q$-period $\sigma^{2}(q)$ would be $q$ times of the variance of 1-period $\sigma^{2}(1)$ [2]. $\sigma^{2}(q)$ and $\sigma^{2}(1)$ are calculated as follows:

$$
\begin{gathered}
\sigma^{2}(q)=\frac{1}{m} \sum_{t=q}^{n q}\left(Y_{t}-Y_{t-q}-q \hat{\mu}\right)^{2} \\
\sigma^{2}(1)=\frac{1}{n q-1} \sum_{t=1}^{n q}\left(Y_{t}-Y_{t-1}-\hat{\mu}\right)^{2} \\
\operatorname{VR}(q)=\frac{\sigma^{2}(q)}{\sigma^{2}(1)}
\end{gathered}
$$

where $m=q(n q-q+1)\left(1-\frac{q}{n q}\right)$ and $\hat{\mu}=\frac{1}{n q}\left(Y_{n q}-Y_{0}\right) . Y_{0}$ and $Y_{n q}$ are $\log c_{0}$ and $\log c_{n q}$, respectively. $\operatorname{VR}(q)$ is the variance ratio.

This paper deploys the variance-ratio test presented in the work of Liu and $\mathrm{He}$ [19], which develops two types of statistics. The first statistic value $z(q)$ is 
Table 5. Result of ADF test on intra-day returns.

\begin{tabular}{ccc}
\hline Model & t-statistic & p-value \\
\hline Level & & \\
without trend & -1.973771 & 0.2986 \\
with trend & -3.41058 & 0.0505 \\
First & & \\
without trend & -31.63682 & $<0.0001$ \\
with trend & -31.62466 & $<0.0001$ \\
\hline
\end{tabular}

developed under the hypothesis of homoskedasticity, where

$$
\begin{gathered}
\phi(q)=\frac{2(2 q-1)(q-1)}{3 q(n q)} . \\
z(q)=\frac{V R(q)-1}{[\phi(q)]^{\frac{1}{2}}} \sim N(0,1)
\end{gathered}
$$

The second statistic value $z^{*}(q)$ is robust to heteroskedasticity:

$$
z^{*}(q)=\frac{V R(q)-1}{\left[\phi^{*}(q)\right]^{\frac{1}{2}}} \sim N(0,1)
$$

where $\phi^{*}(q)=\sum_{j=1}^{q-1}\left[\frac{2(q-1)}{q}\right]^{2} \hat{\delta}(j)$ and

$\hat{\delta}(j)=\frac{\sum_{t=j+1}^{n q}\left(Y_{t}-Y_{t-1}-\hat{\mu}\right)^{2}\left(Y_{t-j}-Y_{t-j-1}-\hat{\mu}\right)^{2}}{\left[\sum_{t=1}^{n q}\left(Y_{t}-Y_{t-1}-\hat{\mu}\right)^{2}\right]^{2}}$.

Lags of $q=2, q=4, q=8$ and $q=16$ days are tested in this study. Table 6 shows the estimates of the homoskedasticity and heteroskedasticity variance-ratio tests and the $z$-statistics. The RWH is rejected for all of the lags in both homoskedasticity and heteroskedasticity statistics. All of the $z$-statistics are statistically lower than -6 , indicating the lack of evidence of mean reversion in the series.

In the test of intra-day returns, time series $Y_{t}$ takes $\log \hat{c}_{d}$. Table 7 shows the results. All of the $z$-statistics are slightly smaller than the corresponding cell of the inter-day result, which suggests that the null hypothesis is rejected and intra-day prices are less random than inter-day prices.

\subsection{Variant of Variance-Ratio Test}

This section extends the idea of the variance-ratio test. The volatilities computed from high frequency prices and daily prices are compared. Their features are unique because the high-frequency prices are at a tiny granularity.

\subsubsection{Features of High-Frequency Data}

Figure 1 illustrates the relationship of the security price and bid-ask spread. In a 
Table 6. Results of Lo and Mac variance-ratio test on inter-day returns.

\begin{tabular}{ccccc}
\hline$q$ (days) & 2 & 4 & 8 & 16 \\
\hline$V R(q)$ & 0.491 & 0.2422 & 0.1243 & 0.0656 \\
$\phi(q)$ & 0.001 & 0.0038 & 0.0095 & 0.021 \\
$z(q)$ & -15.4721 & -12.3116 & -8.99896 & -6.453 \\
$\phi^{*}(q)$ & 0.0003 & 0.0017 & 0.0055 & 0.0134 \\
$z^{*}(q)$ & -30.9624 & -18.5105 & -11.7548 & -8.0657 \\
\hline
\end{tabular}

Table 7. Results of Lo and Mac variance-ratio test on intra-day returns.

\begin{tabular}{ccccc}
\hline$q$ (days) & 2 & 4 & 8 & 16 \\
\hline$V R(q)$ & 0.4864 & 0.2389 & 0.1213 & 0.0607 \\
$\phi(q)$ & 0.0011 & 0.0038 & 0.0095 & 0.0210 \\
$z(q)$ & -15.6132 & -12.3666 & -9.0296 & -6.4866 \\
$\phi^{*}(q)$ & 0.0002 & 0.0013 & 0.0046 & 0.0115 \\
$z^{*}(q)$ & -34.6845 & -20.7901 & -12.9067 & -8.7582 \\
\hline
\end{tabular}

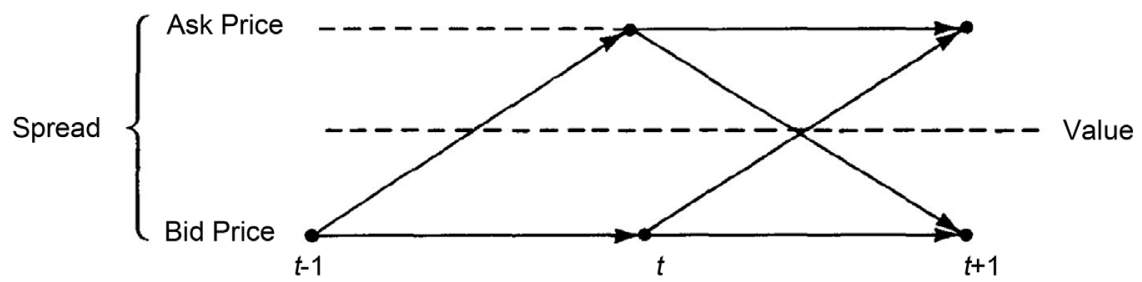

Figure 1. Relationship between security price and bid-ask spread.

short period, the bid 1 and ask1 prices do not change. In Figure 1, two joint directional arrows present one possible path of the security price, and the horizontal dashed lines (from top to bottom) present the ask1 price (denoted as $A$ ), the intrinsic value of the security, and the bid 1 price (denoted as B), respectively. The assumption is that the possibilities of a security price proceeding to bid 1 or ask1 in the next time point is $50 \%-50 \%$. Eight possible paths for the security price subsequently emerge, as depicted in Figure $1^{2}$, namely, $(A, A, A)$, $(A, A, B), \quad A, B, A,(A, B, B),(B, A, A),(B, A, B), B, B, A$ and $(B, B, B)$. If the spread is defined as $s=A-B$, then the corresponding price changes between every two consecutive ticks $\left(\Delta p_{t}, \Delta p_{t+1}\right)$ of the aforementioned eight paths would be $(0,0),(0,-s),(-s, s),(s, 0),(s, 0),(s,-s),(0, s)$ and $(0,0)$.

The covariance of price changes would be

$\operatorname{Cov}\left(\Delta p_{t}, \Delta p_{t+1}\right)=\frac{1}{8}\left(0+0-s^{2}+0+0-s^{2}+0+0\right)=-\frac{s^{2}}{4}$, which does not equal 0.

However, from a macro perspective, such a covariance should be 0 in a random

${ }^{2}$ The figure is copied from Roll [20]. 
process. Generally, the bid1- and ask1-prices are random walks, and the series of closing prices at ticks is formed by selecting either bid1- or ask1-price each time. When the tick is short (i.e., a half-second in the China stock market), the series of high-frequency stock prices does not follow a random walk, although daily closing prices might follow a random walk or the deviation from a random walk is small and therefore it is ignored. To conclude, when statistically investigating high-frequency prices, either bid1- or ask1-prices should be used, instead of closing prices. This paper uses bid1-prices.

\subsubsection{Welch's T-Test}

Originally, the daily volatility $\sigma$ can be estimated by daily data. For a data set of $D(D>1)$ days, an estimate of $\sigma$ would be

$$
\hat{\sigma}_{D}=\sqrt{\frac{\sum_{d=1}^{D}\left(r_{d}-\bar{r}\right)^{2}}{D-1}}
$$

where $r_{d}$ and $\bar{r}$ are daily returns on day $d$ and average returns over $D$ days, respectively. The expectation and variance of $\hat{\sigma}_{D}^{2}$ are as follows:

$$
\begin{gathered}
\exp \left[\hat{\sigma}_{D}^{2}\right]=\sigma^{2} \\
\operatorname{var}\left(\hat{\sigma}_{D}^{2}\right)=\frac{2 \sigma^{4}}{D-1}
\end{gathered}
$$

The null hypothesis is that the bid1-price follows a random walk. Under this hypothesis, $\sigma$ can be estimated by high frequency data. The tick and one day are denoted by $\tau$ and $T=N \tau \quad(T \gg \tau)$, respectively; the tick and daily return volatilities $\delta$ and $\sigma$ subsequently satisfy:

$$
\sigma=\sqrt{\frac{T}{\tau}} \delta
$$

The tick returns volatility $\delta$ can be estimated by

$$
\hat{\delta}=\sqrt{\frac{\sum_{i=1}^{N}\left(b_{i}-\bar{b}\right)^{2}}{N-1}}
$$

where $b_{i}$ pertains to the returns of bid1 price at the ith tick. Consequently, $\sigma$ estimated by Equation (2) and (3) is as follows:

$$
\hat{\sigma}_{H}=\sqrt{\frac{T}{\tau}} \hat{\delta}
$$

Based on the preceding equation, it has

$$
\begin{gathered}
\exp \left[\hat{\sigma}_{H}^{2}\right]=\frac{T}{\tau} \exp \left[\hat{\delta}^{2}\right]=\frac{T}{\tau} \delta^{2}=\sigma^{2} \\
\operatorname{var}\left(\hat{\sigma}_{H}^{2}\right)=\frac{T^{2}}{\tau^{2}} \operatorname{var}\left(\hat{\delta}^{2}\right)=\frac{2 \sigma^{4}}{N-1}
\end{gathered}
$$

In the real market data, $\tau$ is only $500 \mathrm{~ms}$, and $T$ is 270 minutes. Thus $N=32400$. $D$ typically takes 30 days or one year (i.e., $D=30$ or $D=250$ ). 
Therefore, $N \gg D$. If the stock price follows a random walk, then the estimate by high frequency data $\hat{\sigma}_{H}$ is better than that by daily data, in that the former has a smaller variance.

Finally, Welch's t-test is applied. Welch's test is an improvement of the student's $t$-test, and this test is used to verify the hypothesis that two populations have equal means but have possibly unequal variances [21].

Welch's $t$-test is defined by the following formula:

$$
t=\frac{\bar{X}_{1}-\bar{X}_{2}}{\sqrt{\frac{s_{1}^{2}}{N_{1}}+\frac{s_{2}^{2}}{N_{2}}}}
$$

where $\bar{X}_{i}, s_{i}^{2}$ and $N_{i}$ are the $i$ th sample mean, sample variance, and sample size, respectively. The degree of freedom is calculated by the Welch-Statterh Waite equation:

$$
v \approx \frac{\left(\frac{s_{1}^{2}}{N_{1}}+\frac{s_{2}^{2}}{N_{2}}\right)^{2}}{\frac{s_{1}^{4}}{N_{1}^{2} v_{1}}+\frac{s_{2}^{4}}{N_{2}^{2} v_{2}}}
$$

where $v_{i}=N_{i}-1$ is the degree of freedom associated with the $i$ th variance estimate.

In this paper, $\hat{\sigma}_{D}$ are computed in a rolling manner with $D=30$, and $\hat{\sigma}_{H}$ is computed every trading day. The statistical results are summarized in Table 8 .

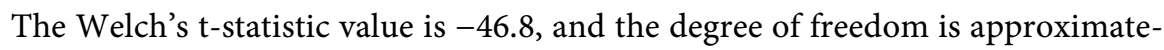
ly equal to 1794 . The $t$-distribution converges to a normal distribution because the degree of freedom is relatively large. Based on the characteristic of a normal distribution, a t-statistic value of -46.8 corresponds to a p-value of less than $1 \mathrm{e}-10$. Thus, the null hypothesis is rejected, and the market is non-random.

\section{Discussion}

The five tests investigate the random of returns from different viewpoints. Jarque-Bera examines the kurtosis and skewness of the curve of returns. Runs test focuses on the direction of changes instead of the specific value of changes. $\mathrm{ADF}$ test verifies the existence of a unit root from the perspective of time series. Variance-ratio test and its variant check the relationship of variances.

Table 8. Results of the Welch's test.

\begin{tabular}{cccc}
\hline & Mean & Standard deviation & Sample size \\
\hline$\hat{\sigma}_{D}$ & 0.01405 & 0.004012 & 893 \\
$\hat{\sigma}_{H}$ & 0.02256 & 0.003728 & \\
\hline Difference & $-37.72 \%$ & \\
t-statistic & -46.8 & \\
$v$ & 1794 & \\
\hline
\end{tabular}


Not all of the aforementioned tests can reject the randomness of the index futures market of China. However, given that some tests reject the randomness, the China market does deviate from the normality to a certain extent. The reasons for such a deviation are beyond the scope of this work; nevertheless, the characteristics of the China market provide several indications.

First, the financial system is unsound. Financial activities are frequently led by policies, such as the crash due to the circuit breaker in 2016. Individual investors are the most active players in the market [22]. The financial products, especially derivatives, are new. The first option was traded in 2015. Second, the legal system is unsound [23]. The Securities and Exchange Commission (SEC) primarily enforces laws and regulates the industry; however, it also has interest of conflict with other parties, such as (local) governments and securities companies. Third, SEC supervision is inadequate. Information disclosure by public companies is incorrect, incomplete, and lacking in timeliness. The delisting mechanism of poorly performing companies is unsound. The SEC currently pays more attention to the listing application than any other stages. Once the company is listed, supervision slackens. Fourth, market participants misbehave. Individual investors are blind, whereas institutional investors manipulate the markets. Moreover, some financial practitioners use legal loopholes.

Resolving the inefficiency entails four aspects. First, government can eliminate unnecessary control over the market and return the freedom to the market. This process can be conducted in stages. The market can be enlivened by encouraging and helping professional talents with overseas experience to work in China, devising various derivatives, and rationally introducing foreign investors. Second, a sound legal system is required. Government should establish a complete legal system, enhance laws, protect the interests of investors, and severely punish lawbreakers. Third, SEC should improve the supervision level and clear delisting conditions. Any activity of a company during the listing should be monitored. Fourth, the government can devote efforts to educating investors and changing the investor composition. Moreover, it should encourage the development of funds to allow individual investors to identify a better means of investing than managing capital by themselves.

\section{Summary}

This paper verifies the normality of the index futures market of China through five statistical tests. Both inter-day and intra-day returns are used. In the literature, intra-day returns are seldom used in statistical tests. The five tests are revised to suit the characteristics of intra-day returns. The fifth test, which is newly proposed, is based on the idea that volatilities calculated from high-frequency and daily data should be consistent if the market is random. However, the Welch's t-test shows that the two volatilities are inconsistent, indicating the abnormality of the market.

In the Jarque-Bera test, single variance-ratio test, and variant of variance-ratio 
test, the normal hypothesis of the index futures market of China is rejected with $5 \%$ confidence; meanwhile, in runs and ADF tests, the randomness cannot be rejected. In particular, the Jarque-Bera statistics for intra-day and inter-day returns are 261.213 and 234.996, respectively. Although both types of returns reject the hypothesis of normal distribution with a confidence of $99.99 \%$, the value of inter-day returns is approximately $10 \%$ less than that of intra-day returns, which suggests that the intra-day market is less random than the overnight market. This phenomenon indicates that the news (information) digestion during market closing increases the transparency of the market, and the daily opening price is as important as the daily closing price.

\section{Acknowledgements}

This research was partially supported by the National Natural Science Foundation of China [grant number 71602109]; Shanghai Pujiang Program [grant number 16PJC038]; and Funding Program for Teachers of Shanghai High Education [grant number ZZSD15095].

\section{References}

[1] Fama, E.F. (1970) Efficient Capital Markets: A Review of Theory and Empirical Work. The Journal of Finance, 25, 383-417. https://doi.org/10.2307/2325486

[2] Lo, A.W. and MacKinlay, A.C. (1988) Stock Market Prices Do Not Follow Random Walks: Evidence from a Simple Specification Test. The Review of Financial Studies, 1, 41-66. https://doi.org/10.1093/rfs/1.1.41

[3] Buguk, C. and Brorsen, B.W. (2003) Testing Weak-Form Market Efficiency: Evidence from the Istanbul Stock Exchange. International Review of Financial Analysis, 12, 579-590. https://doi.org/10.1016/S1057-5219(03)00065-6

[4] Araujo Lima, E.J. and. Tabak, B.M. (2004) Tests of the Random Walk Hypothesis for Equity Markets: Evidence from China, Hong Kong and Singapore. Applied Economics Letters, 11, 255-258. https://doi.org/10.1080/13504850410001674911

[5] Al-Khazali, O.M., Ding, D.K. and Pyun, C.S. (2007) A New Variance Ratio Test of Random Walk in Emerging Markets: A Revisit. Financial Review, 42, 303.-317. https://doi.org/10.1111/j.1540-6288.2007.00173.x

[6] Borges, M.R. (2008) Efficient Market Hypothesis in European Stock Markets. Technical Report, ISEG-School of Economics and Management, Department of Economics, University of Lisbon, Lisbon.

[7] Borges, M.R. (2011) Random Walk Tests for the Lisbon Stock Market. Applied Economics, 43, 631-639. https://doi.org/10.1080/00036840802584935

[8] Malkiel, B.G. (2005) Reflections on the Efficient Market Hypothesis: 30 Years Later. Financial Review, 40, 1-9. https://doi.org/10.1111/j.0732-8516.2005.00090.x

[9] von Gersdorff, N. and Bacon, F. (2009) U.S. Mergers and Acquisitions: A Test of Market Efficiency. Journal of Finance and Accountancy, 1, 1-8.

[10] Gallagher, L.A. and Taylor, M.P. (2002) Permanent and Temporary Components of Stock Prices: Evidence from Assessing Macroeconomic Shocks. Southern Economic Journal, 69, 345-362. https://doi.org/10.2307/1061676

[11] Balachandher, K.G., Fauzias, M.N. and Lai, M.M. (2002) An Examination of the Random Walk Model and Technical Trading Rules in the Malaysian Stock Market. 
Quarterly Journal of Business \& Economics, 41, 81-104.

[12] Hoque, H.A., Kim, J.H. and Pyun, C.S. (2007) A Comparison of Variance Ratio Tests of Random Walk: A Case of Asian Emerging Stock Markets. International Review of Economics \& Finance, 16, 488-502. https://doi.org/10.1016/j.iref.2006.01.001

[13] Balsara, N.J., Chen, G. and Lin, Z. (2007) The Chinese Stock Market: An Examination of the Random Walk Model and Technical Trading Rules. Quarterly Journal of Business \& Economics, 46, 43-63.

[14] Lim, K.J.S., Chadha, P., Lau, J. and Potdar, N. (2012) Is the Mongolian Equity Market Efficient? Empirical Evidence from Tests of Weak-Form Efficiency. Journal of Money, Investment and Banking, 25, 181-193.

[15] Azad, A.S., Azmat, S., Fang, V. and Edirisuriya, P. (2014) Unchecked Manipulations, Price-Volume Relationship and Market Efficiency: Evidence from Emerging Markets. Research in International Business and Finance, 30, 51-71. https://doi.org/10.1016/j.ribaf.2013.05.003

[16] Huang, W., Nakamori, Y. and Wang, S.-Y. (2005) Forecasting Stock Market Movement Direction with Support Vector Machine. Computers \& Operations Research, 32, 2513-2522. https://doi.org/10.1016/j.cor.2004.03.016

[17] Peng, L. and Xiong, W. (2006) Investor Attention, Overconfidence and Category Learning. Journal of Financial Economics, 80, 563-602. https://doi.org/10.1016/j.jfineco.2005.05.003

[18] Qian, B. and Rasheed, K. (2007) Stock Market Prediction with Multiple Classifiers. Applied Intelligence, 26, 25-33. https://doi.org/10.1007/s10489-006-0001-7

[19] Liu, C.Y. and He, J. (1991) A Variance-Ratio Test of Random Walks in Foreign Exchange Rates. The Journal of Finance, 46, 773-785. https://doi.org/10.1111/j.1540-6261.1991.tb02686.x

[20] Roll, R. (1984) A Simple Implicit Measure of the Effective Bid-Ask Spread in an Efficient Market. The Journal of Finance, 39, 1127-1139. https://doi.org/10.1111/j.1540-6261.1984.tb03897.x

[21] Welch, B.L. (1947) The Generalization of "Student's" Problem when Several Different Population Variances Are Involved. Biometrika, 34, 28-35.

[22] Bailey, W., Cai, J., Cheung, Y.L. and Wang, F. (2009) Stock Returns, Order Imbalances, and Commonality: Evidence on Individual, Institutional, and Proprietary Investors in China. Journal of Banking \& Finance, 33, 9-19. https://doi.org/10.1016/j.jbankfin.2006.08.007

[23] Lee, A.K. (2015) China's Economy, the Hidden Truths: China's Economy Seen in the Undercurrent of Organized Unaccountability. CreateSpace Independent Publishing, Charleston. 